\title{
Práticas de autocuidado do idoso com diabetes mellitus em um município amazônico
}

\author{
Self-care practices of the elderly with diabetes mellitus in an amazon municipality \\ Prácticas de autocuidado de los ancianos con diabetes mellito en un municipio amazónico \\ Stéfany Caetano Corrêa ${ }^{1 *}$, Deyvylan Araujo Reis ${ }^{1}$.
}

\begin{abstract}
RESUMO
Objetivo: Analisar as práticas de autocuidado e nível de dependência dos idosos com Diabetes Mellitus (DM) cadastrados em uma Unidade Básica de Saúde (UBS) em um município Amazônico. Métodos: Estudo descritivo, transversal e quantitativo, composto de pessoas com idade igual ou superior a 60 anos, ambos os sexos, cadastrados na (UBS). Aplicaram-se quatro instrumentos: Formulário sociodemográfico; Questionário de Atividades de Autocuidado com o Diabetes (QAD); Índice de Katz e Escala de Lawton. Os dados coletados foram registrados e o tratamento foi feito pelo uso de estatística descritiva, tendo como auxilio o programa Statistical Package for the Sciences (SPSS) na versão 20.0. Resultados: Constatou-se que o nível de dependência nas Atividades Instrumentais da Vida Diária (AIVDs) se correlacionou predominantemente na variável idade (50\%) entre a faixa etária de 60 a 69 anos de idade quando comparada aos idosos da faixa etária de 70 a 79 anos. Nas Atividades Básicas da Vida Diária (ABVDs), sua máxima deu-se para independentes para todas as faixas etárias. Conclusão: Averiguo-se que os idosos com DM desenvolvem as práticas de autocuidado de forma independente em sua pluralidade. As ABVDs apresentaram-se independentemente $(75 \%)$, em contraste com os dados apresentados pelas AIVDs desempenhadas pelos idosos, demonstrando-se dependentes (75\%).
\end{abstract}

Palavras-chave: Autocuidado, Idosos, Diabetes mellitus.

\begin{abstract}
Objective: To analyze the self-care practices of the elderly with Diabetes Mellitus (DM) registered in a Basic Health Unit (BHU) in an Amazon Municipality. Methods: Descriptive, cross-sectional and quantitative study, composed of people $\geq 60$ years, both sexes registered at the (BHU). Four instruments were applied: form sociodemographic; index of Katz and Lawton Scale. The collected were recorded statistics, using the Statistical Package for the program Sciences (SPSS in version 20.0). Results: It was found that the level of dependence in Instrumental Activities of Daily Living (IADLs) correlated predominantly in the variable age (50\%) between the age group of 60 to 69 year of age when compared to the elderly of the age group 70 to 79 years old. In Basic Activities of Daily Living (BADLs), it maxim was given to independent for all age group. Conclusion: It was found that the elderly with DM develop self-care practices independently in their plurality. (BADLs) presented independently (75\%) in contrast to the data presented by the (IADLs) performed by the elderly, showing dependence (75\%).
\end{abstract}

Keywords: Self-care, Elderly, Diabetes mellitus.

1 Universidade Federal do Amazonas (UFAM), Coari - AM. *E-mail: stefanypietro@hotmail.com

Fundação de Amparo à Pesquisa do Estado do Amazonas - FAPEAM. PIB-S/0183/2017.

SUBMETIDO EM: 5/2020

ACEITO EM: 6/2020

PUBLICADO EM: 8/2020

REAS/EJCH | Vol.12(10) | e3847 | DOI: https://doi.org/10.25248/reas.e3847.2020 Página 1 de 11 


\section{RESUMEN}

Objetivo: Analizar las prácticas de autocuidado y el nivel de dependencia de los ancianos com Diabetes Mellitus (DM) registrada em una Unidad Básica de Salud (UBS) en un Municipio Amazonico. Métodos: Estudio descriptivo, transversal y cuantitativo, compuesto por personas de 60 años o más, ambos sexos, registradas en (UBS). Se aplicaron cuatro instrumentos: forma sociodemográfica; Cuestionario de actividades de autocuidado de la diabetes (PAF); Índice de Katz y escala de Lawton. Los datos recopilados se registraron y el tratamiento se realizó mediante estadística descriptiva, utilizando el programa Paquete estadístico para las ciencias (SPSS) en la versión 20.0. Resultados: Se encontró que el nivel de dependencia de Las Actividades Instrumentales de la Vida Diaria (AIVDs) se correlacionaron en la variable edad $(50 \%)$ entre el grupo de edad 60 a 69 años en compariación con los acianos del grupo de edad de 70 a 70 años. En Actividades Basicas de la Vida Diaria ABVDs, su máxima se le dio a los independientes para todos los grupos de edad. Conclusión: Se encuentra que los ancianos con (DM) desarrollar prácticas de autocuidado de forma (75\%), en contraste con los datos presentados por el AIVDs realizado por los ancianos, que muestran dependencias $(75 \%)$.

Palabras clave: Autocuidado, Ancianos, Diabetes mellitus.

\section{INTRODUÇÃO}

O crescimento da população idosa brasileira conduz a uma reestruturação dos grupos etários, que não significa apenas uma alteração demográfica, mas também uma transformação no sistema de saúde, uma vez que esse aumento requer a necessidade de garantir a esse público, além de mais anos de vida, um atendimento voltado às peculiaridades que o envolvem.

Devido a isso, percebeu-se um aumento gradativo no surgimento de Doenças Crônicas Não Transmissíveis (DCNTs), consideradas no mundo as mais recorrentes causas de mortalidade (PIUVEZAM G, et al., 2016; MENDES ACG, et al., 2012).

Entre as DCNTs, o Diabetes Mellitus (DM) é uma comorbidade que, na maioria das vezes, se agrega a estilo de vida impróprio, que se associam com fatores genéticos preexistentes, alterações fisiológicas do processo de envelhecimento e fatores de riscos modificáveis como o consumo de alimentos inadequados para a saúde, à obesidade e o sedentarismo. Sabe-se que há tipos distintos de DM; a classificação proposta pela Organização Mundial da Saúde (OMS) e pela Associação Americana de Diabetes (AAD) inclui quatro classes clínicas DM1, DM2, DM gestacional e DM associado a outras condições, sendo que estes variam quanto a etiologia, evolução clínica e tratamento (CHEEVER KH, et al., 2016; SDB, 2015).

No ano de 2016, no Estado do Amazonas (AM), as internações no Sistema Único de Saúde (SUS) correspondiam a 134.169, sendo que 15.884 foram de pessoas acima de 60 anos de idade, o que pode estar relacionado ao acelerado envelhecimento da população, com maior tendência ao sedentarismo e a hábitos alimentares indevidos, além de outras mudanças sociais e comportamentais, que contribuem para os crescentes níveis de incidência e prevalência da DM.

Nessa perspectiva, o idoso com DM deve ser informado sobre a importância do autocuidado para um tratamento efetivo. É necessário conscientizar os idosos portadores dessa enfermidade quanto à sua condição, orientando-os, juntamente com sua família e equipe de saúde. Deve-se buscar um trabalho com mais eficiência no controle da hiperglicemia e controle dos agravos, motivando esses idosos a adotarem um estilo de vida adequado (VIANNA MS, et al., 2017; SILVA 2016; SBD, 2016).

Os aspectos mencionados, a carência de estudos científicos e a importância da pesquisa envolvendo idosos com DM justificam a relevância social e acadêmica desta investigação. Além disso, esta contribui para o aperfeiçoamento da temática, pois permite conhecer como se desenvolvem as práticas de autocuidado de idosos com DM no contexto domiciliar. 
O objetivo da pesquisa é analisar as práticas de autocuidado dos idosos e o nível de dependência dos portadores de DM cadastrados em uma Unidade Básica de Saúde (UBS) do município de Coari, Estado do Amazonas. Buscando nortear este estudo, estabeleceu-se a seguinte questão de pesquisa: "Quais são as práticas de autocuidado de idosos portadores de Diabetes Mellitus em uma UBS em um município Amazônico?".

\section{MÉTODOS}

Estudo descritivo e transversal, com abordagem quantitativa. A população estudada é composta de indivíduos com idade igual ou superior a 60 anos, de ambos os sexos, cadastrados e residentes na área de abrangência da UBS. O cenário do estudo foi uma UBS da área urbana no município, e o período de realização da pesquisa foi de agosto de 2017 a junho de 2018. A amostra foi constituída por 20 idosos portadores de DM, cadastrados no Sistema de Acompanhamento de Hipertensão e Diabetes (HIPERDIA).

Os critérios de inclusão do estudo foram: idosos 60 anos ou mais com diagnóstico médico de DM apontado no cadastro do HIPERDIA, de ambos os sexos, que apresentassem condições para responder às perguntas contidas nos instrumentos de coleta de dados e aceitassem participar voluntariamente da pesquisa, por meio da assinatura do Termo de Consentimento Livre e Esclarecido (TCLE). Foram excluídos do estudo aqueles que não atendiam aos critérios de inclusão.

Os dados foram coletados por meio de quatro instrumentos: $1^{\circ}$ ) Formulário sociodemográfico construído para este estudo, utilizando e adaptando questões do questionário da Amostra por Domicílio do Censo Demográfico do ano de 2010, do Instituto Brasileiro de Geografia e Estatística (IBGE); 2º) Questionário de Atividades de Autocuidado com o Diabetes (QAD), sendo este um instrumento traduzido e adaptado para o Brasil; 3) O Índice de Katz, instrumento que avalia as sete Atividades Básicas de Vida Diária (ABVDs) e 4º) Escala de Lawton, que avalia o desempenho funcional do indivíduo nas nove Atividades Instrumentais da Vida Diária (AIVDs).

Neste estudo, foram utilizados questionários validados e confiáveis com a finalidade de proporcionar autoconhecimento e contribuir para intervenções adequadas para a coleta de dados nas entrevistas com os participantes da pesquisa. Esse tipo de instrumento é recomendado pelo Ministério da Saúde (MS), na versão validada brasileira, por ser considerado confiável (BRASIL, 2006).

Os dados e as informações coletadas foram registrados em uma planilha do programa Microsoft Excel 2010, utilizando o programa Statistical Package for the Sciences (SPSS) versão 20.0 para a análise estatística. Na apresentação dos dados, foi realizada uma análise descritiva, por meio das variáveis do estudo, por distribuição de frequência, porcentagem e medidas descritivas.

Este estudo atendeu rigorosamente à Resolução no 466/2012 do Conselho Nacional de Saúde e foi aprovado pelo Comitê de Ética e Pesquisa da UFAM sob o no CAAE: 67022217.60000 .5020 e parecer ํo 2.107.085.

\section{RESULTADO E DISCUSSÂO}

A média de idade entre os 20 participantes do estudo, em anos completos, foi de 69,3 ( $d p=11,32$ ). A idade mínima analisada é de 60 anos e a máxima de 99 anos. Destaca-se que a média para o sexo feminino é de $67(\mathrm{dp}=12)$ e para o masculino 71 anos $(\mathrm{dp}=11)$. A faixa etária mais frequente observada foi a de 60 a 69 anos (70\%).

Os valores, em média, encontrados em outras pesquisas mostram de 68 a 73 anos de idade de idoso com Diabetes Mellitus (VICENTE MC, et al., 2019; GONÇALVES LHTR, et al., 2020; PRADO MAMB, et., 2016). Os dados estão representados na Tabela 1. 
Tabela 1 - Distribuição dos idosos com Diabetes Mellitus, conforme as variáveis sociodemográficas e condição de saúde, 2018.

\begin{tabular}{ccc}
\hline Variáveis & N & $\%$ \\
\hline Faixa etária & 14 & 70 \\
60 a 69 & 2 & 10 \\
70 a 79 & 4 & 20 \\
$\geq 80$ & 10 & 50 \\
Sexo & 10 & 50 \\
\hline Masculino & & 45 \\
Feminino & 9 & 30 \\
\hline Estado conjugal & 6 & 25 \\
\hline Casados/União estável & 5 & \\
Viúvos & & 60 \\
Solteiros/Divorciados & 12 & \\
\hline Escolaridade & 8 & 70 \\
\hline Ensino fundamental incompleto & & 30 \\
\hline Religabetos & 14 & 85 \\
\hline Evangélicos & 6 & 15 \\
\hline Católicos & & 55 \\
\hline Renda pessoal & 17 & 35 \\
\hline 1 & 3 & 10 \\
\hline$\geq 1$ & 11 & 100 \\
\hline Número de morbidades & 7 &
\end{tabular}

Fonte: Corrêa SC e Reis DA, 2020.

Verificou-se que ambos os sexos apresentaram o percentual de $(50 \%)$ cada. Estudos apontam que um maior número de pessoas do sexo feminino tem sido diagnosticado com Diabetes Mellitus. A Pesquisa Nacional de Saúde (PNS), concretizada em 2013 constatou uma maior incidência de DM em mulheres em comparação aos homens (IBGE, 2014).

Diferentemente do que foi encontrado neste estudo, em que ambos os sexos apresentaram o mesmo percentual com relação à doença. Referente ao estado conjugal, a maioria dos indivíduos deste estudo são casados/união estável (45\%), seguidos de viúvos (30\%) e solteiros/divorciados (25\%).

Nesse sentido, esses dados assemelham-se aos encontrados por Goulart JCM (2013) em um estudo com 50 pessoas diagnosticadas com DM2, cadastradas numa Estratégia de Saúde da Família (ESF), em ItajubáMG, em que (46\%) dos participantes do estudo eram casados, (36\%) viúvos e (18\%) separados/solteiros.

No que tange à escolaridade, houve predominância no baixo nível de escolaridade, sendo que 12 (60\%) com ensino fundamental incompleto e oito (40\%) eram analfabetos. Esses dados refletem o aspecto brasileiro na educação, que influenciam na adesão ao tratamento, compreensão da doença e cuidados. Resultados são similares a um estudo com 66 idosos com (DM2), registrados em uma (UBS) na cidade de Maringá (PR).

Nesta investigação, constataram que a maioria tinha baixo nível de escolaridade $(37,88 \%)$ eram analfabetos, $(30,3 \%)$ com ensino fundamental incompleto, $(16,67 \%)$ concluíram o ensino fundamental $(12,12 \%)$ e $(3,03 \%)$ cursaram o ensino médio e o ensino superior, respectivamente (CAROLINO IDR, et al., 2008).

Quanto à religião, a pesquisa aponta que a mais frequente na distribuição apresentada foi à categoria evangélica (70\%), seguida da católica (30\%). Resultados que difere da pesquisa de Vicente MC, et al. (2019) com 98 idosos com DM, eram representados por católico (72,9\%). No que se refere à renda pessoal, a maioria (85\%) dos idosos entrevistados afirmou receber até um salário mínimo. Esses dados são análogos aos encontrados por Marques MB, et al. (2013), em estudo realizado com cem idosos com diagnóstico de DM, 
em Centros de Saúde da Família em Fortaleza (CE), que observou que a maioria $(36,0 \%)$ dos portadores de DM do tipo 2 recebiam de um a dois salários mínimos.

Referente às outras morbidades, $20(100 \%)$ referiram outras patologias, na sua maioria para duas comorbidades representados por 11 idosos (55\%). Alusivo às outras comorbidades a mais evidentes nos portadores de DM foram as Doenças do Sistema Circulatório (DSCs), como a Hipertensão Arterial Sistêmica (HAS) com (75\%), e as doenças nutricionais/metabólica, como a dislipidemia (25\%), definida como distúrbio que altera os níveis séricos dos lipídeos (gorduras). Tais resultados vão ao encontro do que afirma o Ministério da Saúde, que aponta como frequente a associação entre dislipidemia e hipertensão arterial (BRASIL, 2006).

A Sociedade Brasileira de Cardiologia (SBC) destaca que a HAS é uma problemática de saúde de maior incidência na atualidade e aumenta com a idade. Essa doença é prevalente na população adulta, no sexo masculino, sendo mais frequente em pessoas com idade de 60 a 69 anos (50\%) e naquelas com 70 anos ou mais $(75 \%)$.

Considerando que as doenças cardiovasculares podem ter o mesmo componente genético e ambiental que o DM, comprova-se a hipótese da agregação da HAS com a DM (MCLELLAN KCP, et al., 2007). Esses achados se confirmam neste estudo, pois a HAS estava presente em $(75 \%)$ dos indivíduos diabéticos entrevistados, o que vem corroborar com o resultado da pesquisa que encontraram maior percentual referida pelos idosos diabéticos a HAS (SBC, 2016; PRADO MAMB, et al., 2016).

Quanto à ocupação dos idosos no presente estudo, obteve-se predomínio de aposentados (95\%), apenas um (5\%) referiu não receber qualquer benefício. Esses dados são congruentes com os achados do estudo de prevalência de idosos com DM realizado no interior do Estado de São Paulo, com 52 portadores da doença, realizado em 2006, que também identificaram uma proporção maior de $(68,5 \%)$ aposentados (OTERO ML, et al., 2007).

Sobre a autoavaliação da condição de saúde, a variável que foi mais citada pelos 20 idosos (100\%) foi "regular" por 14 (70\%) participantes, seguida de "boa" por três (15\%). Da mesma forma se mostraram os resultados quando feito um comparativo da variável sexo com a variável "saúde autorreferida", quando se observou que ambos os sexos referiram à sua saúde como "regular" $(70 \%)$ e, com relação às demais variáveis, na maioria, os homens declararam sua saúde como "boa" (15\%), seguidos das mulheres, que a avaliaram como "ruim" (15\%) respectivamente. Estudo de Prado MAMB, et al. (2016) constataram que os idosos com duas ou mais doenças crônicas, associada com a DM tinham percepção da sua saúde como boa (69\%), sendo diferente ao encontrado neste estudo. Os dados estão representados no Gráfico 1.

Gráfico 1 - Distribuição dos idosos com Diabetes Mellitus, segundo a sua autoavaliação da condição de saúde, 2018.

\section{Autoavaliação da condição de saúde}

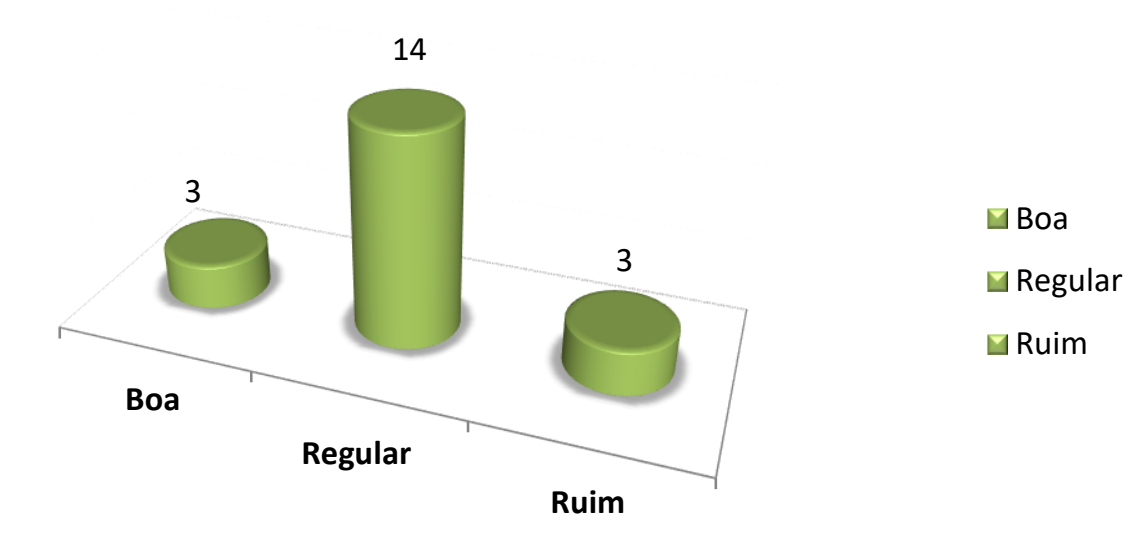

Fonte: Corrêa SC e Reis DA, 2020. 
No que se refere à adesão às práticas de autocuidado, verificou-se que os participantes relataram com maior frequência como: "seguir uma dieta saudável" (80\%) e "secar os espaços entre os dedos dos pés depois de lavá-los" ( $80 \%)$, com média de $5,75(\mathrm{dp}=1,61)$ para ambos; "examinar dentro dos sapatos antes de calçalos" relatados pelos 14 participantes $(70 \%)$, com média de $4,70(\mathrm{dp}=3,21)$.Importa registrar que todos os participantes do estudo informaram que não consumia doces representado em (100\%) na frequência de zero a quatro dias da semana, com média de 0,60 dias $(\mathrm{dp}=0,99)$.

Esses dados são semelhantes encontrados no estudo de Vicente MT, et al. (2019) e divergem da investigação realizada em Ribeirão Preto, cujos resultados apontaram para o maior consumo de doces (COELHO ACM, 2013). A avaliação dos itens do Questionário de Atividades de Autocuidado (QAD) pelos participantes do estudo está descrita na Tabela 2.

Na dimensão alimentação geral, especificamente a "alimentação saudável realizada nos últimos sete dias", os participantes informaram "seguir uma dieta saudável entre cinco a sete dias" (80\%). Tal achado também foi constatado em um estudo realizado em Ribeirão Preto (SP), no ano de 2013, o qual encontrou uma média de 5,0 dias de realização de dieta saudável comparada ao segundo item do QAD de 3,74, que é "seguir dieta com orientação profissional" (GOMIDES DS, et al., 2013).

Os resultados observados para essa variável estão em consonância com os da pesquisa de Freitas MCF, et al. (2012), que estimou 96 sujeitos diagnosticados com DM, cadastrados na Estratégia de Saúde da Família (ESF) do município de Teresina (PI), em que a maior aderência foi "comer cinco ou mais porções de frutas ou vegetais" e a menor foi "seguir as orientações nutricionais".

Estudo de Vicente MT, et al. (2019), que constatou maior média para o item da alimentação geral do QAD, com média de $6,43(+1,22)$, o que vem corroborar com esta pesquisa. Com relação a dimensão dos cuidados com os pés, a variável "secar os espaços entre os dedos dos pés depois de lavá-los" foi bem evidenciada pelos participantes $(80 \%)$.

A compreensão do idoso sobre a gravidade do problema e a importância da realização de ações de autocuidado pode ser crucial na prevenção ou retardo do aparecimento de resultados adversos. O cuidado com os pés configura-se como uma atitude simples, mas determinante na qualidade de vida dos idosos portadores de DM.

Para os idosos que não tinham adesão às atividades do QAD foram: "avaliar o açúcar no sangue o número de vezes recomendado pelos profissionais de saúde" (90\%), "tomar injeções de insulina conforme recomendado" (85\%) e "realizar exercícios físicos específicos" (85\%). Com relação à automonitorização da glicemia em número de dias, essa medida é essencial no controle do Diabetes Mellitus (Gomides DS, et al. 2013).

No presente estudo, a atividade obteve média de 0,95 ( $\mathrm{dp}=0,95)$, com frequência de 18 (90\%) afirmaram realizá-la entre zero a quatro dias. Nos escores do questionário $Q A D$, é considerada distante do desejável, que é sete. 
Tabela 2 - Avaliação dos itens do Questionário de Atividades de Autocuidado (QAD) com Diabetes pelos participantes do estudo, 2018.

\begin{tabular}{|c|c|c|c|}
\hline \multirow{3}{*}{ Dimensão } & \multicolumn{2}{|c|}{ Frequência (dias) } & \multirow{3}{*}{$\begin{array}{c}\text { Média } \\
\left(\mathrm{dp}^{\star}\right)\end{array}$} \\
\hline & 0 a 4 & 5 a 7 & \\
\hline & $\mathbf{N}(\%)$ & $\mathbf{N}(\%)$ & \\
\hline \multicolumn{4}{|l|}{ Alimentação geral } \\
\hline Seguir uma dieta saudável & $4(20)$ & $16(80)$ & $5,75(1,61)$ \\
\hline Seguir a orientação alimentar de um profissional & $11(55)$ & $9(45)$ & $3,95(2,58)$ \\
\hline \multicolumn{4}{|l|}{ Alimentação específica } \\
\hline Consumir cinco ou mais porções de frutas e/ou vegetais & $10(50)$ & $10(50)$ & $3,85(2,81)$ \\
\hline $\begin{array}{l}\text { Consumir carne vermelha e ou derivados de leite } \\
\text { integral }\end{array}$ & $17(85)$ & $3(15)$ & $1,80(2,39)$ \\
\hline Consumir doces & $20(100)$ & - & $0,60(0,99)$ \\
\hline \multicolumn{4}{|l|}{ Atividade física } \\
\hline Realizar atividade física, pelo menos 30 minutos & $16(80)$ & $4(20)$ & $1,75(2,86)$ \\
\hline Realizar exercício físico específico & $17(85)$ & $3(15)$ & $1,40(2,56)$ \\
\hline \multicolumn{4}{|l|}{ Monitorização da glicemia } \\
\hline Avaliar o açúcar no sangue & $17(85)$ & $3(15)$ & $1,10(2,07)$ \\
\hline $\begin{array}{l}\text { Avaliar o açúcar no sangue o número de vezes } \\
\text { recomendado pelo profissional de saúde }\end{array}$ & $18(90)$ & $2(10)$ & $0,95(1,93)$ \\
\hline \multicolumn{4}{|l|}{ Cuidados com os pés } \\
\hline Examinar os pés & $8(40)$ & $12(60)$ & $4,50(3,25)$ \\
\hline Examinar dentro dos sapatos antes de calçá-los & $6(30)$ & $14(70)$ & $4,70(3,21)$ \\
\hline $\begin{array}{l}\text { Secar os espaços entre os dedos dos pés depois de } \\
\text { lavá-los }\end{array}$ & $4(20)$ & $16(80)$ & $5,75(2,33)$ \\
\hline \multicolumn{4}{|l|}{ Medicação } \\
\hline $\begin{array}{l}\text { Tomar medicamentos do diabetes conforme } \\
\text { recomendado }\end{array}$ & $8(40)$ & $12(60)$ & $4,35(3,23)$ \\
\hline Tomar injeções de insulina conforme recomendado & $17(85)$ & $3(15)$ & $1,05(2,56)$ \\
\hline Tomar número indicado de comprimidos para diabetes & $10(50)$ & $10(50)$ & $3,55(3,26)$ \\
\hline
\end{tabular}

Legenda: (-) corresponde a zero. *Nota: o instrumento de QAD é parametrizado em dias por semana, de 0 a 7 dias, sendo zero a situação menos desejável, e sete mais favoráveis para as atividades de autocuidado. Neste estudo, o escore obtido entre 0 e 4 dias corresponde a não adesão às práticas de autocuidado, enquanto o intervalo entre 5 e 7 dias está relacionado à adesão. Essa situação é diferente para as dimensões alimentação específica (consumir carne vermelha, leites e derivados e doces), em que o resultado do valor é invertido. *dp= Desvio-padrão.

Fonte: Corrêa SC e Reis DA, 2020.

No seguimento a prática de "realização de exercício físico", o estudo mostrou que a aderência é muito baixa, tendo em média de 1,40 ( $\mathrm{dp}=2,56$ ) dias na semana, que vem corroborar com o estudo de Gomides DS, et al. (2012), no qual verificou-se uma média de 1,0 dia por semana para a realização de exercícios físicos. De acordo com Michels MJ, et al. (2010) na American College of Sport Medicine, para uma boa qualidade de vida é necessária uma quantidade mínima de 150 minutos por semana de atividades físicas.

Quanto ao tabagismo, neste estudo, o índice de fumantes há mais de dois anos apresentou-se predominantemente baixo, onde a maioria dos participantes (90\%) relatou não fumar e apenas (10\%) afirmaram ser fumantes. Esses dados divergem de outro estudo em que se observou o predomínio (80\%) de participantes que referiram não ter fumado um cigarro, mesmo que uma tragada, durante os últimos sete dias (MARQUES MB, et al., 2013).

Na presente pesquisa, ainda que tenha sido pequeno o número de indivíduos que relataram não ter fumado nos sete dias anteriores, cabe ressaltar que se devem fomentar ações que estimulem o combate ao tabagismo. A importância de tais ações é apontada em pesquisas que afirmam que há relação entre o uso do 
tabaco e o aumento do número de amputações e complicações em portadores de Diabetes Mellitus (GOMIDES DS, et al., 2013).

A maior frequência evidenciada neste estudo foi para independência nas ABVDs. As ABVDs com grau de dependência, que recebem assistência parcial foram as variáveis "banho" $(20 \%)$ e o "uso do banheiro" (10\%). No grau de dependência nas ABVDs, nas que recebem "assistência total", observou-se atividades como: "vestir-se" (10\%) e "continência" (5\%). Conforme Moraes EN (2012), explica que em virtude do decaimento funcional, no desempenho das ABVDs, ocorre uma sequência previsível e hierárquica como: banhar-se, vestir-se, usar o banheiro, transferir-se, ter continência e alimentar-se. Ramos LR, et al. (2013) indicam, através de uma análise fatorial, que as ABVDs que, irão representar um alto grau de dependência em idosos são: "levantar da cama", "tomar banho" e "andar".

Quadro 1 - Distribuição dos idosos com Diabetes Mellitus, conforme o grau de dependência pela classificação das ABVDs no Índice de Katz e AIVDs na Escala de Lawton, 2018.

\begin{tabular}{|l|c|c|c|}
\hline \multicolumn{1}{|c|}{ ABVDs } & $\begin{array}{c}\text { Não recebe } \\
\text { assistência } \\
\mathbf{N}(\%)\end{array}$ & $\begin{array}{c}\text { Recebe assistência } \\
\text { parcial } \\
\mathbf{N}(\%)\end{array}$ & $\begin{array}{c}\text { Recebe assistência } \\
\text { total } \\
\mathbf{N}(\%)\end{array}$ \\
\hline Banho & $16(80)$ & $4(20)$ & - \\
\hline Vestuário & $17(85)$ & $1(5)$ & $2(10)$ \\
\hline Uso do banheiro & $18(90)$ & $2(10)$ & - \\
\hline Transferência & $20(100)$ & - & - \\
\hline Continência & $19(95)$ & - & - \\
\hline Alimentação & $19(95)$ & $1(5)$ & Necessita de ajuda \\
total \\
\hline
\end{tabular}

Legenda: (-) corresponde a zero. Fonte: Corrêa SC e Reis DA, 2020.

$\mathrm{Na}$ escala de Lawton, as atividades realizadas com maior frequência com independência, que correspondem ao requesito "sem ajuda", foram: "conseguir usar o telefone" (65\%) e "conseguir ir a locais distantes" (65\%). Já as AIVDs com maior relato de dependência (com ajuda parcial ou total) foram "conseguir tomar remédio com dose e horário certos" (45\%), "conseguir cuidar de suas finanças" (45\%), "fazer compras" (40\%) e "arrumar a casa" (40\%). Tal achado está em conformidade com os resultados de uma pesquisa realizada em Goiânia, na qual $(58,1 \%)$ dos participantes apresentaram algum grau de dependência para AIVDs (NUNES DP, et al., 2010).

Verificou-se que, dos 20 idosos entrevistados para index de ABVDs, 15 (75\%) eram independentes para todas as atividades e três (15\%) eram independentes para todas as atividades, exceto para uma. Não houve nenhum participante do estudo categorizado como totalmente dependente. A classificão do índice de Katz e Escala de Lawton dos idosos com DM é apresentada na Tabela 3. 
Tabela 3 - Distribuição dos idosos com Diabetes Mellitus, conforme a classificação do índice de Katz e a Escala de Lawton, 2018.

\begin{tabular}{lcc}
\multicolumn{1}{c}{ Index de ABVDs (Katz) } & N & $\%$ \\
\hline $\begin{array}{l}\text { A- Independente para todas as atividades } \\
\text { B- Independente para todas as atividades, menos uma }\end{array}$ & 15 & 75 \\
$\begin{array}{l}\text { C- Independente para todas as atividades, menos banhar-se, vestir-se e uma } \\
\text { adicional }\end{array}$ & 3 & 15 \\
$\begin{array}{l}\text { D- Independente para todas as atividades, menos banhar-se, vestir-se, ir ao } \\
\text { banheiro e uma adicional. }\end{array}$ & 1 & 5 \\
\hline \multicolumn{1}{c}{ Escore de Lawton } & 5 \\
\hline Independente & 5 & 25 \\
$\begin{array}{l}\text { Dependente parcial } \\
\text { Dependente total }\end{array}$ & 15 & 75 \\
\hline
\end{tabular}

Legenda: (-) corresponde a zero. Nota: ${ }^{*}$ Classificação conforme index of independence in Activities of Daily Living de Katz.

Fonte: Corrêa SC e Reis DA, 2020.

No tange aos dados obtidos sobre o escore de Lawton dos idosos com Diabetes Mellitus, constatou-se que $(75 \%)$ dos participantes do estudo eram dependentes para AIVDs. Esse resultado pode ser explicado pelo fato de as Atividades Instrumentais da Vida Diária (AIVDs) necessitar de maior desenvolvimento da parte física e cognitiva, maior desenvoltura nas relações sociais e a capacidade de lidar com as adversidades (FREITAS MCF, et al., 2012; TORRES GV, et al., 2010).

Constatou-se que o nível de dependência nas AIVDs apresentou em sua maioria relação com a idade $(50 \%)$ entre as "faixas etárias de 60 a 69 anos de idade", quando comparados aos idosos da "faixa de 70 a 79 anos". E, quanto às ABVDs, as referidas faixas etárias se mostraram independentes. A análise e a distribuição das variáveis sociodemográficas, condição de saúde com o grau de dependência nas Atividades Básicas da Vida Diária (ABVDs) e Atividades Instrumentais da Vida Diária (AIVDs) dos idosos participantes do estudo está apresenta na Tabela 4.

Quanto ao nível de independência para as AIVDs no autocuidado, a faixa etária de 60 a 69 anos apresentou o maior percentual representado pelas atividades conseguir usar o telefone $(65 \%)$ e ir a locais distantes (65\%). Esse achado difere do resultado de estudo realizado na zona urbana na cidade de Pelotas, em que cerca de $(43 \%)$ dos idosos apresentaram dificuldade para utilizar o meio de transporte (DEL DUCA GF, et al., 2009).

A variável "sexo masculino" apresentou-se independente (50\%) para a capacidade funcional de ABVDs; já o "sexo feminino" apresentou um maior grau de dependência (40\%) para AIVDs. Quanto à "escolaridade", os idosos com o "ensino fundamental incompleto" apresentaram maior percentual (45\%) de dependência para AIVDs.

No que diz respeito à "renda pessoal", verificou-se que os idosos com renda de "um salário mínimo" apresentaram o maior quantitativo $(85 \%)$ de independência para ABVDs. No que se refere ao "número de comorbidades", o maior percentual deu-se para até "dois tipos de doenças" (50\%), apresentando um grau maior de capacidade funcional de independência para ABVDs.

Portanto, entende-se que idosos com idade mais avançada, com renda mensal referente até um salário mínimo, e nível de escolaridade relativamente baixo são os mais propícios à perda da capacidade funcional, pois, quanto menos recursos financeiro e menor o ensino, mais tendem a se distanciar do restante do meio em que convivem e da sociedade em geral.

Neste caso necessita-se analisar primariamente as dificuldades de entendimento e a distância dos núcleos de referência para eles. Desta maneira, essa quantidade populacional carece de assistência integral, não somente por parte de específicos profissionais de saúde, mas também de uma equipe multiprofissional, haja vista que a capacidade funcional envolve múltiplas dimensões, como financeira, educacional e social (PINTO $\mathrm{AH}$, et al., 2016). 
Tabela 4 - Distribuição das variáveis sociodemográficas, condição de saúde com grau de dependência nas ABVDs e AIVDs dos idosos com Diabetes Mellitus, 2018.

\begin{tabular}{|c|c|c|c|c|}
\hline \multirow{4}{*}{ Variáveis } & \multicolumn{4}{|c|}{ Capacidade Funcional } \\
\hline & \multicolumn{2}{|c|}{ ABVDs } & \multicolumn{2}{|c|}{ AIVDs } \\
\hline & IND & DEP & IND & DEP \\
\hline & $\mathbf{N}(\%)$ & N (\%) & $\mathbf{N}(\%)$ & $\mathbf{N}(\%)$ \\
\hline \multicolumn{5}{|l|}{ Faixa etária } \\
\hline 60 a 69 & $14(70)$ & - & $4(20)$ & $10(50)$ \\
\hline 70 a 79 & $2(10)$ & - & $1(5)$ & $1(5)$ \\
\hline$\geq 80$ & $3(15)$ & $1(5)$ & - & $4(20)$ \\
\hline \multicolumn{5}{|l|}{ Sexo } \\
\hline Feminino & $9(45)$ & $1(5)$ & $3(15)$ & $7(35)$ \\
\hline Masculino & $10(50)$ & - & $2(10)$ & $8(40)$ \\
\hline \multicolumn{5}{|l|}{ Escolaridade } \\
\hline Ensino fundamental incompleto & $11(55)$ & $1(5)$ & $3(15)$ & $7(35)$ \\
\hline Analfabetos & $8(40)$ & - & $2(10)$ & $8(40)$ \\
\hline \multicolumn{5}{|l|}{ Renda pessoal (SM) ${ }^{\star}$} \\
\hline$<1$ & $17(85)$ & - & $5(25)$ & $12(60)$ \\
\hline$\geq 1$ & $2(10)$ & $1(5)$ & - & $3(15)$ \\
\hline \multicolumn{5}{|l|}{ Número de morbidades } \\
\hline 2 & $10(50)$ & $1(5)$ & $4(20)$ & $7(35)$ \\
\hline 3 & $7(35)$ & - & $1(5)$ & $6(30)$ \\
\hline 4 & $2(10)$ & - & - & $2(10)$ \\
\hline
\end{tabular}

Legenda: (-) corresponde a zero ; ( $\left.{ }^{*}\right)$ salário mínimo vigente no ano de 2018 no valor de $R \$ 954,00$. Nota: a faixa etária dos idosos foi determinada segundo Veras $R$ (2009), que os classifica em jovem idoso (60 a 69 anos), meio idoso (70 a 79 anos) e idoso (acima de 80 anos).

Fonte: Corrêa SC e Reis DA, 2020.

\section{CONCLUSÃO}

Conclui-se no presente estudo que não houve predomínio quanto à variável sexo, ambos apresentaram o mesmo quantitativo na amostra, a maioria eram na faixa etária de 60-69 anos, casados, com nível socioeconômico e educativo baixo. Constatou-se ainda que os idosos desenvolvem o seu próprio autocuidado no tratamento da DM. Os idosos pesquisados apresentaram capacidade funcional para as Atividades da Vida Diária, tanto para básicas quanto para as instrumentais. Porem com relação ao Questionário de Atividades de Autocuidado (QAD) foi identificado que os idosos têm baixa adesão em algumas práticas nos dias da semana. Quanto ao desempenho das (ABVDs) e (AIVDs), percebeu-se que, na sua maioria, os idosos eram funcionalmente independentes nas (ABVDs) com (75\%), não requerendo apoio por parte de cuidadores e/ou familiares, diferentemente do que se apresentou quanto às (AIVDs) com quantitativo semelhante de (75\%), para as quais necessitam de algum auxílio no ambiente domiciliar e fora dele. As variáveis independentes, idade renda e escolaridade influenciam evidentemente na manutenção da qualidade funcional. Por fim, espera-se com os resultados obtidos nesta investigação que possam servir para contribuir e ampliar o conhecimento sobre os fatores de autocuidado assim como os fatores sóciodemográficos, socioeconômico, clínicos de saúde e terapêuticos, além de verificar as atividades no desenvolvimento de autocuidado dos idosos portadores de DM.

\section{REFERÊNCIAS}

1. BRASIL. Ministério da Saúde. Secretaria de Atenção à Saúde. Departamento de Atenção Básica. Envelhecimento e saúde da pessoa idosa. (Caderno de Atenção Básica no19). Ministério da Saúde, 2006.

2. BRASIL. Ministério da Saúde. Secretaria de Atenção à Saúde. Departamento de Atenção Básica. Hipertensão arterial sistêmica para o Sistema Único de Saúde. (Caderno de Atenção Básica no16). Brasília: Ministério da Saúde, 2006b. 
3. CAROLINO IDR, et al. Fatores de risco em pacientes com diabetes mellitus tipo 2, Ribeirão Preto. Rev. Latino-Am. Enfermagem 2008; 16(2): 238-244.

4. CHEEVER KH, et al. Tratado de Enfermagem Médica-Cirúrgica. 13nd ed. Rio de Janeiro: Guanabara Koogan, 2016.

5. COELHO ACM. Autocuidado das pessoas com diabetes mellitus tipo 2 em seguimento ambulatorial. Dissertação (Mestrado em Ciências, Programa Enfermagem Fundamental) - Escola de Enfermagem de Ribeirão Preto. Universidade de São Paulo, Ribeirão Preto, 2013; 112p.

6. DEL DUCA GF, et al. Incapacidade funcional para atividades básicas e instrumentais da vida diária em idosos. Rev Saúde Pública, 2009; 43(5):796-805.

7. FREITAS MCF, et al. Relação entre apoio social, adesão aos tratamentos e controle metabólico de pessoas com diabetes mellitus. Rev Latino-Am Enfermagem. 2012; 20(1): 52-8.

8. GOMIDES DS, et al. Self-care of people with diabetes mellitus who have lower limb complications. Acta paul. enferm. 2013; 26 (3):289-93.

9. GONÇALVES LHT, et al. Conhecimento e atitude sobre diabetes mellitus de usuários idosos com a doença atendidos em unidade básica de saúde. Revista Nursing.2020;23(260):3497-3501.

10. GOULART JCM. Avaliação das ações de autocuidado da pessoa portadora de diabetes mellitus. Monografia (Graduação em Enfermagem) - Escola de Enfermagem Wenceslau Brás, Itajubá, Minas Gerais, 2013; 80p.

11. IBGE-Instituto Brasileiro de Geografia e Estatística. Diretoria de Pesquisas, Coordenação de População e Indicadores Sociais, Projeção da População do Brasil e Unidades da Federação por sexo e Idade para o período de 2000-2030. 2013.

12. IBGE-Instituto Brasileiro de Geografia e Estatística. Ministério da. Secretaria do Planejamento, Orçamento e Gestão. Pesquisa Nacional de Saúde 2013. Brasília: Ministério da Saúde, 2014.

13. MARQUES MB, et al. Avaliação da competência de idosos diabéticos para o autocuidado. São Paulo. Rev. esc. enferm, 2013; 47(2): 415-420.

14. MCLELLAN KCP, et al. Diabetes mellitus do tipo 2, síndrome metabólica e modificação no estilo de vida. Rev.Nutr. Campinas, 2007 Outubro; 20(5): 515-524.

15. MENDES ACG, et al. Assistência Pública de Saúde no Contexto da Transição Demográfica Brasileira: exigências atuais e futuras. Cad.Saúde Pública, 2012; 28(5):955-964.

16. MICHELS MJ, et al. Questionário de Atividades de Autocuidado com o Diabetes: tradução, adaptação e avaliação das propriedades psicométricas. Arq. Bras. Endocrinol Metab, 2010; 54(7):644-651.

17. MORAES EN. Atenção à saúde do idoso: aspectos conceituais. Brasília: Organização Pan Americana da Saúde, 2012; 98p.

18. NUNES DP, et al. Capacidade funcional, condições socieconômicas e de saúde de idosos atendidos por equipes de Saúde da Família de Goiânia (GO-Brasil). Cien. saude coletiva, 2010; 15(6): 2872-2898.

19. OTERO ML, et al. Características sociodemográficas e clínicas de portadores de diabetes em um serviço de atenção básica à saúde. Rev.lat.-am.enferm, 2007 Outubro; 15(spe): 768-773.

20. PINTO AH, et al. Capacidade funcional para atividades da vida diária de idosos da Estratégia Saúde da Família da zona rural.Rev Ciencias e Saúde Coletiva, 2016; 21(11): 9-14.

21. PIUVEZAM G, et al. Atenção Primaria a Saúde e os Idosos Institucionalizados: a perspectiva da gestão Municipal no Brasil. Rev. Port. de Saúde Pública, 2016; 34(1): 92-100.

22. PRADO MAMB, et al. Diabetes em idosos: uso de medicamentos e risco de interação medicamentosa. Ciência \& Saúde Coletiva, 2016; 21(11):3447-3458.

23. RAMOS LR, et al. Perguntas mínimas para rastrear dependência em atividades da vida diária em idosos. Rev.Saude Publica, 2013; 47(3);506-13.

24. SBC-Sociedade Brasileira de Cardiologia. VI Diretrizes Brasileira de Hipertensão. Vol 107, n 3, sup. 3. São Paulo, 2016.

25. SBD-Sociedade Brasileira de Diabetes. Diretrizes da Sociedade Brasileira de Diabetes (2015-2016). AC Farmacêutica. São Paulo, 2016.

26. SILVA KR. Prevalência de síndrome da fragilidade em idosos da área urbana do município de Coari-Amazonas: Um estudo de base populacional. Dissertação (Mestrado em saúde, sociedade e endemias). Universidade Federal do Amazonas, Manaus-Amazonas, 2016; 84p.

27. Torres GV, et al. Avaliação da capacidade funcional em idosos residentes em área periférica do interior da Bahia / Nordeste do Brasil. Arq. Neuro-Psiquiatr. Fev 2010; 68 (1): 39-43.

28. VERAS R. Envelhecimento populacional contemporâneo: demandas, desafios e inovações. Revista de Saúde Pública, 2009. 43(3): 548-554.

29. VIANNA MS, et al. Self-carecompetence in theadministration of insulin in olderpeopleaged 70 orover. Rev. Latino-Am. Enfermagem. 2017; 2(25): e2943.

30. VICENTE MT, et al. Resiliência e autocuidado de pessoas idosas com diabetes mellitus. Rev Rene. 2019;20:e33947. 